\title{
Review of: "Patterns of medication errors involving pediatric population reported to the French Medication Error Guichet"
}

\author{
Patricia Darbishire ${ }^{1}$ \\ 1 Purdue University
}

Potential competing interests: The author(s) declared that no potential competing interests exist.

Overall impression: A well designed, well written manuscript. The authors might consider emphasizing the comparison of the types of adult and pediatric medication errors, as well as the different settings in the title, as these aspects are what makes the article unique, and a significant contribution to a well published topic. A sufficient number of relevant references were included. Their literature search was thorough. The study objectives are clearly described in the introduction section and the content is relevant to the practice of pharmacy and to an international audience. The authors have explored some new and important aspects of medication safety. Likewise, the authors thoroughly and accurately made comparisons of their findings to others' previously published literature. I saw no major methodological flaws in the study design. The data gathering and use of statistical analysis were both appropriate. Conclusions are clear and supported by the primary results of the study. The tables and figures are relevant and provide sufficient details, although they are somewhat redundant with the text. The implications of this research include how and where we might make system changes to reduce medication errors - a potentially high impact on practice. Overall, a significant contribution to the literature, with no major study flaws - therefore, I give it 5 stars. 